\title{
Existence of the Mild Solution for Impulsive Semilinear Differential Equation
}

\author{
Alka Chadha and Dwijendra N. Pandey \\ Department of Mathematics, Indian Institute of Technology Roorkee, Roorkee, Uttarakhand 247667, India \\ Correspondence should be addressed to Alka Chadha; alkachaddha03@gmail.com
}

Received 26 December 2013; Revised 15 April 2014; Accepted 18 April 2014; Published 18 May 2014

Academic Editor: Yuri N. Skiba

Copyright (c) 2014 A. Chadha and D. N. Pandey. This is an open access article distributed under the Creative Commons Attribution License, which permits unrestricted use, distribution, and reproduction in any medium, provided the original work is properly cited.

\begin{abstract}
We study the existence of solutions of impulsive semilinear differential equation in a Banach space $X$ in which impulsive condition is not instantaneous. We establish the existence of a mild solution by using the Hausdorff measure of noncompactness and a fixed point theorem for the convex power condensing operator.
\end{abstract}

\section{Introduction}

In a few decades, impulsive differential equations have received much attention of researchers mainly due to its demonstrated applications in widespread fields of science and engineering such as biology, physics, control theory, population dynamics, medicine and so on. The real world processes and phenomena which are subjected during their development to short-term external inuences can be modeled as impulsive differential equation. Their duration is negligible compared to the total duration of the entire process or phenomena. Impulsive differential equations are an appropriate model to hereditary phenomena for which a delay argument arises in the modelling equations. To further study on impulsive differential equations, we refer to books $[1,2]$ and papers [3-11].

In this paper, our purpose is to establish the existence of a solution to the following differential equations with non instantaneous impulses

$$
\begin{gathered}
u^{\prime}(t)=-A u(t)+F(t, u(t)), \quad t \in\left(s_{i}, t_{i+1}\right], \\
i=0,1, \ldots, \delta, \\
u(t)=G_{i}(t, u(t)), \quad t \in\left(t_{i}, s_{i}\right], i=1, \ldots, \delta, \delta \in \mathbb{N}, \\
u(0)=x_{0},
\end{gathered}
$$

where $-A: D(A) \subset X \rightarrow X$ is a closed and bounded linear operator with dense domain $D(A) \subset X$. We assume that $-A$ is the infinitesimal generator of a strongly continuous semigroup $\{\mathscr{T}(t), t \geq 0\}$ in a Banach space $(X,\|\cdot\|)$. Here, $x_{0} \in X, 0=t_{0}=s_{0}<t_{1} \leq s_{1} \leq t_{2}<\cdots<t_{\delta} \leq s_{\delta}<t_{\delta+1}=T_{0}$ and $F:\left[0, T_{0}\right] \times X \rightarrow X, G_{i}:\left(t_{i}, s_{i}\right] \times X \rightarrow X$ for all $i=1, \ldots, \delta$ are suitable functions to be specified later.

In [4], authors have introduced a new class of abstract impulsive differential equations in which impulses are not instantaneous and established the existence of solutions to the problem (1)-(3) with the assumption that operator $A$ generates a $C_{0}$-semigroup of bounded linear operators. In this system of (1)-(3), the impulses begin all of a sudden at the points $t_{i}$ and their proceeding continues on a finite time interval $\left[t_{i}, s_{i}\right]$ [5]. To concern the hemodynamical harmony of an individual we think about the following simplified situation. One can recommend a few intravenous sedates (insulin) on account of a decompensation (e.g., high or low level of glucose). Since the presentation of the medications in the bloodstream and the ensuing retention for the form are progressive and continuous processes, we can depict this circumstance as an impulsive activity which begins abruptly and stays animated on a finite time interval.

In [12], the generalization of the condensing operator as convex-power condensing operator has been introduced by Sun and Zhang and a new fixed point theorem for convexpower condensing operator has been established. The new fixed point theorem for convex-power condensing operator, defined by Sun and Zhang, is the generalization of the famous Schauder's fixed point theorem and Sadovskii's fixed point 
theorem. Sun and Zhang [12] have considered the following problem:

$$
\begin{gathered}
u^{\prime}(t)+A u(t)=f(t, u(t)), \quad 0 \leq t \leq T_{0}, \\
u(0)=x_{0}
\end{gathered}
$$

in a Banach space and established existence theorems for positive mild solutions and global mild solutions to the problem (4) with noncompact semigroup. The nonlinear function $f$ fulfills the suitable conditions on the measure of noncompactness as $\alpha(f(t, B(t))) \leq \mathscr{L} \alpha(B(t))$ for any bounded set $B \subset X$, where $\alpha$ is the Kuratowski measure of noncompactness and $\mathscr{L}$ is a positive constant. For more details about measure of noncompactness, we refer to [1220].

In the present work, our aim is to obtain results concerning the existence of mild solutions to problem (1)-(3) by using convex-power condensing operator and fixed point theorem for convex-power condensing operator Sun and Zhang [12].

The organization of the paper is as follows. We provide some basic definitions, Lemmas and theorems in Section 2 as "preliminaries." We prove the existence of a mild solution for system (1) in Section 3 as "existence of mild solution." In the last section, we present an example to illustrate the application of the abstract results.

\section{Preliminaries}

In this section, we give some definitions, notations, theorems, and lemmas which will be used in later sections.

Let $(X,\|\cdot\|)$ be a real Banach space. The symbol $C([0$, $\left.\left.T_{0}\right] ; X\right)$ stands for the Banach space of all the continuous functions from $\left[0, T_{0}\right]$ into $X$ equipped with the norm $\|z\|=$ $\sup \left\{\|z(s)\|, s \in\left[0, T_{0}\right]\right\}$ and $L^{1}\left(\left[0, T_{0}\right], X\right)$ stands for the space of $X$-valued Bochner integrable functions on $\left[0, T_{0}\right]$ endowed with the norm $\|f\|_{L^{1}}=\int_{0}^{T_{0}}\|f(t)\| d t, f \in L^{1}\left(\left[0, T_{0}\right], X\right)$.

The operator $-A: D(A) \subset X \rightarrow X$ is the infinitesimal generator of a uniformly continuous semigroup $\{\mathscr{T}(t), t \geq 0\}$ and $D(A)$ denotes the domain of $A$, which is densely defined, endowed with the graph norm. A semigroup $\mathscr{T}(t)$ is said to be equicontinuous if $\{\mathscr{T}(t) y: y \in K\}$ is equicontinuous at arbitrary $t, 0<t<\infty$, for any bounded subset $K \subset X$. Throughout the paper we assume that

(H1) the operator $-A$ generates the equicontinuous semigroup $\{\mathscr{T}(t) ; t \geq 0\}$ and there exists a positive number $M$ such that $\|\mathscr{T}(t)\| \leq M$.

For the study of impulsive differential equation, we define the space $\mathscr{P} \mathscr{C}\left(\left[0, T_{0}\right] ; X\right)$ which contains all the functions $y:\left[0, T_{0}\right] \rightarrow X$ such that $y(\cdot)$ is continuous at $t \neq t_{j}$, $y\left(t_{j}^{-}\right)=y\left(t_{j}\right)$ and $y\left(t_{j}^{+}\right)$exists for all $j=1,2, \ldots, \delta$. Clearly, $\mathscr{P} \mathscr{C}\left(\left[0, T_{0}\right] ; X\right)$ is a Banach space endowed with norm $\|y\|_{\mathscr{P C}}=\sup \left\{y(t): t \in\left[0, T_{0}\right]\right\}$. For a function $y \in$ $\mathscr{P} \mathscr{C}\left(\left[0, T_{0}\right] ; X\right)$ and $i \in\{0,1, \ldots, \delta\}$, we define the function $\widetilde{y}_{i} \in C\left(\left[t_{i}, t_{i+1}\right], X\right)$ such that

$$
\widetilde{y}_{i}(t)= \begin{cases}y(t), & \text { for } t \in\left(t_{i}, t_{i+1}\right], \\ y\left(t_{i}^{+}\right), & \text {for } t=t_{i} .\end{cases}
$$

For $B \subset \mathscr{P} \mathscr{C}\left(\left[0, T_{0}\right] ; X\right)$, we have $\widetilde{B}_{i}=\left\{\widetilde{y}_{i}: y \in B\right\}$ and we have following Accoli-Arzelà type criteria.

Lemma 1 (see [4]). A set $B \subset \mathscr{P} \mathscr{C}\left(\left[0, T_{0}\right] ; X\right)$ is relatively compact in $\mathscr{P} \mathscr{C}\left(\left[0, T_{0}\right] ; X\right)$ if and only if each set $\widetilde{B_{j}}(j=0,1$, $\ldots, \delta)$ is relatively compact in $C\left(\left[t_{j}, t_{j+1}\right], X\right)(j=0,1, \ldots, \delta)$.

Now we present the following definition of mild solution.

Definition 2. A piecewise continuous function $u \in \mathscr{P} \mathscr{C}([0$, $\left.\left.T_{0}\right] ; X\right)$ is said to be a mild solution of the (1)-(3) if $u(0)=x_{0}$, $u(t)=G_{i}(t, u(t))$, for all $t \in\left(t_{i}, s_{i}\right], i=1, \ldots, \delta$, and

$$
u(t)=\mathscr{T}(t) x_{0}+\int_{0}^{t} \mathscr{T}(t-\zeta) F(\zeta, u(\zeta)) d \zeta
$$

for all $t \in\left[0, t_{1}\right]$, and

$$
u(t)=\mathscr{T}\left(t-s_{i}\right) G_{i}\left(s_{i}, u\left(s_{i}\right)\right)+\int_{s_{i}}^{t} \mathscr{T}(t-\zeta) F(\zeta, u(\zeta)) d \zeta,
$$

for all $t \in\left[s_{i}, t_{i+1}\right]$ and every $i=1, \ldots, \delta$.

Next, we give the definition of the Hausdorff measure of noncompactness (MNC).

Definition 3 (see [14]). The Hausdorff measure of noncompactness $\beta$ of the set $E$ in Banach space $X$ is the greatest lower bound of those $\varepsilon>0$ for which the set $E$ has in the space $X$ a finite $\varepsilon$-net; that is,

$$
\beta(E)=\inf \{\varepsilon>0: E \text { has a finite } \varepsilon \text {-net in } X\},
$$

for every bounded subset $E$ in a Banach space $X$.

Definition 4 (see [14]). The Kuratowski measure of noncompactness $\alpha$ defined on each bounded subset $E$ of $X$ as

$$
\begin{aligned}
\alpha(E)=\inf \{\epsilon>0: & E \text { is covered by a finite } \\
& \text { number of sets with diameter } \leq \epsilon\} .
\end{aligned}
$$

The relation between Kuratowski measure of noncompactness $\alpha$ and the Hausdorff measure of noncompactness $\beta$ is given by

$$
\beta(E) \leq \alpha(E) \leq 2 \beta(E) .
$$

To set the structure for our primary existence results, we review some essential properties about the Kuratowski and Hausdorff measure of noncompactness.

Lemma 5. For any bounded set $U, V \subset Y$, where $Y$ is a Banach space. Then, we have following results:

(i) $\beta(U)=0$ if and only if $U$ is precompact;

(ii) $\beta(U)=\beta(\operatorname{conv} U)=\beta(\bar{U})$, where $\operatorname{conv} U$ and $\bar{U}$ denote the convex hull and closure of $U$, respectively;

(iii) $\beta(U) \subset \beta(V)$, when $U \subset V$; 
(iv) $\beta(U+V) \leq \beta(U)+\beta(V)$, where $U+V=\{u+v: u \in$ $U, v \in V\}$;

(v) $\beta(U \cup V) \leq \max \{\beta(U), \beta(V)\}$;

(vi) $\beta(\lambda U)=\lambda \cdot \beta(U)$, for any $\lambda \in \mathbb{R}$;

(vii) If the map $\mathbb{Q}: D(\mathbb{Q}) \subset Y \rightarrow \mathscr{Z}$ is continuous and satisfies the Lipschitsz condition with constant $\kappa$, then, we have that $\chi_{\mathscr{E}}(Q U) \leq \kappa \beta(U)$ for any bounded subset $U \subset D(Q)$, where $Y$ and $\mathscr{Z}$ are Banach space.

Definition 6. A continuous and bounded map $Q: D \subseteq X \rightarrow$ $X$ is called $\beta$-condensing if, for any noncompact bounded subset $E \subset D$,

$$
\beta(Q E)<\beta(E),
$$

where $X$ is a Banach space.

To avoid confusion, we denote by $\beta(\cdot)$ the Hausdorff measure of noncompactness on set $X, C\left(\left[0, T_{0}\right] ; X\right)$ and $\mathscr{P} \mathscr{C}\left(\left[0, T_{0}\right] ; X\right)$.

Lemma 7 (see [14], Darbo-Sadovskii). Let $D \subset X$ be bounded, closed, and convex. If the continuous map $Q: D \rightarrow$ $D$ is a $\beta$-contraction, then the map $Q$ has a fixed point in $D$.

In [12], authors have presented the generalization of the condensing operator and suggested a new fixed point theorem for such operators. Firstly, we present some recognition. Let $D \subset X$ be a bounded, closed, and convex set and let $\mathbb{Q}$ be a continuous map from $D$ into itself with $u_{0} \in D$. For every $E \subset D$, we set

$$
\mathbb{Q}^{\left(1, u_{0}\right)}(E)=\mathscr{Q}(E), \quad \widehat{Q}^{\left(n, u_{0}\right)} E=\mathscr{Q}\left(\overline{\operatorname{conv}}\left\{\mathscr{Q}^{n-1, u_{0}} E, u_{0}\right\}\right),
$$

$$
\text { for } n=2,3, \ldots \text {. }
$$

Definition 8 (see [12]). Let $D \subset X$ be bounded, closed, and convex. A bounded and continuous operator $Q$ : $D \rightarrow$ $D$ is called a convex-power condensing operator if for any bounded nonprecompact subset $E \subset D$, there exist $x_{0} \in D$ and an integer $n_{0}>0$ such that

$$
\beta\left(Q^{\left(n_{0}, u_{0}\right)}(E)\right)<\beta(E) .
$$

A $\beta$-convex-power condensing operator is $\beta$-condensing for $n_{0}=1$. Therefore, it is clear that convex-power condensing operator is a generalization of the condensing operator.

Lemma 9 (see [12]). Let $D \subset X$ be bounded, closed, and convex set. If the continuous map $Q: D \rightarrow D$ is $\beta$-convexpower condensing, then there exists a fixed point of map $\mathbb{Q}$ in D.

Lemma 10 (see $[11,14])$. If $E \subseteq C\left(\left[0, T_{0}\right] ; X\right)$ is bounded, then $\beta(E(t)) \leq \beta(E), \forall t \in\left[0, T_{0}\right]$, where $E(t)=\{u(t) ; u \in E\} \subseteq X$. In addition, if $E$ is equicontinuous on $\left[0, T_{0}\right]$, then $\beta(E(t))$ is continuous on $\left[0, T_{0}\right]$ and

$$
\beta(E)=\sup _{t \in\left[0, T_{0}\right]}\{\beta(E(t))\} .
$$

Lemma 11 (see [11]). If $E \subseteq \mathscr{P} \mathscr{C}\left(\left[0, T_{0}\right] ; X\right)$ is bounded, then $\beta(E(t)) \leq \beta(E), \forall t \in\left[0, T_{0}\right]$. Besides, suppose the following conditions are satisfied:

(1) E is equicontinuous on $\left[0, t_{1}\right]$ and each $\left(t_{i}, s_{i}\right],\left[s_{i}, t_{i+1}\right]$, $i=1, \ldots, \delta$,

(2) $E$ is equicontinuous at $t=t_{i}^{+}, i=1, \ldots, \delta$.

Then, we have $\beta(E)=\sup _{t \in\left[0, T_{0}\right]} \beta(E(t))$.

Lemma 12 (see [14]). If $E \subset C\left(\left[0, T_{0}\right] ; X\right)$ is bounded and equicontinuous, then $\beta(E(t))$ is continuous and

$$
\beta\left(\int_{0}^{t} E(\tau) d \tau\right) \leq \int_{0}^{t} \beta(E(\tau)) d \tau, \quad \forall t \in\left[0, T_{0}\right],
$$

where $\int_{0}^{t} E(\tau) d \tau=\left\{\int_{0}^{t} w(\tau) d \tau, w \in E\right\}$

Lemma 13. Let $\left\{u_{n}\right\}_{n=1}^{\infty}$ be a sequence of functions in $L^{1}\left(\left[0, T_{0}\right] ; \mathbb{R}_{+}\right)$. Suppose that there exist $\gamma(t) \in L^{1}\left(\left[0, T_{0}\right] ; \mathbb{R}_{+}\right)$ satisfying $\left\|u_{n}(t)\right\| \leq \gamma(t)$ for almost all $t \in\left[0, T_{0}\right]$ and every $n \geq 1$. Then, we have

$$
\begin{array}{r}
\beta\left(\left\{\int_{0}^{t} u_{n}(\tau) d \tau: n \geq 1\right\}\right) \leq 2 \int_{0}^{t} \beta\left(\left\{u_{n}(\tau)\right\}_{n=1}^{\infty}\right) d \tau \\
\forall t \in\left[0, T_{0}\right] .
\end{array}
$$

Lemma 14. We assume that (H1) holds. Then the set $\left\{\int_{0}^{t} \mathscr{T}(t-\right.$ $\zeta) u(\zeta) d \zeta,\|u(\zeta)\| \leq \eta(\zeta)$ for a.e. $\left.\zeta \in\left[0, T_{0}\right]\right\}$ is equicontinuous for all $t \in\left[0, T_{0}\right]$.

Proof. Let $h$ be a positive constant such that $0 \leq t<t+h \leq T_{0}$. For $u \in \mathscr{P} \mathscr{C}$, we have

$$
\begin{aligned}
& \left\|\int_{0}^{t+h} \mathscr{T}(t+h-\zeta) u(\zeta) d \zeta-\int_{0}^{t} \mathscr{T}(t-\zeta) u(\zeta) d \zeta\right\| \\
& \quad \leq\left\|\int_{0}^{t}[\mathscr{T}(t+h-\zeta)-\mathscr{T}(t-\zeta)] u(\zeta) d \zeta\right\| \\
& \quad+\int_{t}^{t+h}\|\mathscr{T}(t+h-\zeta) u(\zeta) d \zeta\|
\end{aligned}
$$

It is obvious for $t=0$. Let $\epsilon>0$ be arbitary integer with $0<\epsilon<t$. For $t>0$, we have

$$
\begin{aligned}
\| \int_{0}^{t} \mathscr{T}( & +h-\zeta) u(\zeta) d \zeta-\int_{0}^{t} \mathscr{T}(t-\zeta) u(\zeta) d \zeta \| \\
\leq & \| \mathscr{T}(h+\epsilon) \int_{0}^{t-\epsilon} \mathscr{T}(t-\epsilon-\zeta) u(\zeta) d \zeta-\mathscr{T}(\epsilon) \\
& \left.\times \int_{0}^{t-\epsilon} \mathscr{T}(t-\epsilon-\zeta) u(\zeta) d \zeta\right) \| \\
& +\left\|\int_{t-\epsilon}^{t} \mathscr{T}(t+h-\zeta) u(\zeta) d \zeta\right\| \\
& +\left\|\int_{t-\epsilon}^{t} \mathscr{T}(t-\zeta) u(\zeta) d \zeta\right\|
\end{aligned}
$$


Since $\mathscr{T}(t), t>0$ is equicontinuous; therefore,

$$
\left\|[\mathscr{T}(h+\epsilon)-\mathscr{T}(\epsilon)] \int_{0}^{t-\epsilon} \mathscr{T}(t-\epsilon-\zeta) u(\zeta) d \zeta\right\| \longrightarrow 0,
$$

as $h \rightarrow 0$, uniformly for $u$. Second and third terms of (18) tend to zero when $\epsilon \rightarrow 0$ since $\epsilon$ is arbitrary small.

Then from (17), (18), and (19) and the absolute continuity of integrals, we obtain that $\left\{\int_{0}^{t} \mathscr{T}(t-\zeta) u(\zeta) d \zeta,\|u(\zeta)\| \leq \eta(\zeta)\right.$ for a.e. $\left.\zeta \in\left[0, T_{0}\right]\right\}$ is equicontinuous for all $t \in\left[0, T_{0}\right]$.

\section{Main Results}

In this section, the existence of the mild solution of (1)-(3) under some specified conditions on $G_{i}$ is established by using the measure of noncompactness and fixed point theorem for convex-power condensing operator.

Now, we made the following hypothesis which will be useful for proving our results.

(HG) The functions $G_{i}(i=1, \ldots, \delta)$ are compact and continuous.

(HF) $F:[0, T] \times X \rightarrow X$ is a nonlinear function such that $F$ satisfies the Carathèodary condition; that is, we have the following.

(i) $F(\tau, \cdot): X \rightarrow X$ is continuous for a.e. $\tau \in$ $\left[0, T_{0}\right]$.

(ii) For each $x \in X, F(\cdot, x):\left[0, T_{0}\right] \rightarrow X$ is strongly measurable. For any $k>0$, there exists a function $m_{k} \in L^{1}\left(\left[0, T_{0}\right], \mathbb{R}_{+}\right)$such that for $u \in X$

$\|F(s, u)\| \leq m_{k}(s), \quad$ for a.e. $s \in\left[0, T_{0}\right]$.

(iii) There exists a constant $\mathscr{L}>0$ such that for any bounded set $B \subset X$,

$\beta(F(\tau, B)) \leq \mathscr{L} \beta(B), \quad$ for a.e. $\tau \in\left[0, T_{0}\right]$.

(HG1) The functions $G_{i}$ are continuous and there exist positive constants $\mathscr{L}_{G_{i}}$ such that

$$
\left\|G_{i}(\tau, x)-G_{i}(\tau, y)\right\| \leq \mathscr{L}_{G_{i}}\|x-y\|,
$$

for all $x, y \in X, \tau \in\left(t_{i}, s_{i}\right],(i=1, \ldots, \delta)$.

$(\mathrm{Hk})$

$$
\begin{gathered}
\max _{\{i=1, \ldots, \delta\}}\left[\left\|m_{k}\right\|_{L^{1}\left(\left[0, T_{0}\right]\right)}+\sup _{t \in\left[0, T_{0}\right]}\left\|G_{i}(t, u(t))\right\|\right. \\
\left.+\left\|x_{0}\right\|\right] \leq k .
\end{gathered}
$$

(H) $\max _{\{i=1, \ldots, \delta\}} M\left(\mathscr{L}_{G_{i}}+4 \mathscr{L} T_{0}\right)<1$.

Theorem 15. Suppose that (H1), (HG), (HF), and (Hk) are satisfied. Then, there exists at least one mild solution on $\left[0, T_{0}\right]$ for the problem (1)-(3).
Proof. We define the operator $\mathbb{Q}: \mathscr{P} \mathscr{C}\left(\left[0, T_{0}\right] ; X\right) \rightarrow$ $\mathscr{P} \mathscr{C}\left(\left[0, T_{0}\right] ; X\right)$ as $\mathscr{Q} u(0)=x_{0}, \mathscr{Q} u(t)=G_{i}(t, u(t))$, for all $t \in\left(t_{i}, s_{i}\right]$ and

$$
\mathcal{Q} u(t)=\mathscr{T}(t) x_{0}+\int_{0}^{t} \mathscr{T}(t-\zeta) F(\zeta, u(\zeta)) d \zeta, \quad t \in\left[0, t_{1}\right]
$$

for $i=0,1, \ldots, \delta$ and for $t \in\left[s_{i}, t_{i+1}\right]$, where $i=1, \ldots, \delta$,

$$
Q u(t)=\mathscr{T}\left(t-s_{i}\right) G_{i}\left(s_{i}, u\left(s_{i}\right)\right)+\int_{s_{i}}^{t} \mathscr{T}(t-\zeta) F(\zeta, u(\zeta)) d \zeta
$$

To prove the result, we show that the operator $\mathbb{Q}$ has a fixed point. Firstly, we show that the $\mathbb{Q}$ is continuous on $\mathscr{P} \mathscr{C}\left(\left[0, T_{0}\right] ; X\right)$. Let $\left\{u_{n}\right\}_{n=1}^{\infty}$ be a sequence in $\mathscr{P} \mathscr{C}\left(\left[0, T_{0}\right] ; X\right)$ such that $\lim _{n \rightarrow \infty} u_{n}=u$ in $\mathscr{P} \mathscr{C}\left(\left[0, T_{0}\right] ; X\right)$. For $t \in\left[s_{i}, t_{i+1}\right]$, we have

$$
\begin{aligned}
\|\left(Q u_{n}\right) & (t)-(\mathscr{Q} u)(t) \| \\
\leq & M\left\|G_{i}\left(s_{i}, u_{n}\left(s_{i}\right)\right)-G_{i}\left(s_{i}, u\left(s_{i}\right)\right)\right\| \\
& +M \int_{s_{i}}^{t}\left\|F\left(\zeta, u_{n}(\zeta)\right)-F(\zeta, u(\zeta))\right\| d \zeta .
\end{aligned}
$$

By the continuity of $F$ and $G_{i}(i=1 \ldots, \delta)$, we have

$$
\begin{array}{cl}
\lim _{n \rightarrow \infty} F\left(t, u_{n}(t)\right) & =F(t, u(t)), \quad t \in\left[0, T_{0}\right], \\
\lim _{n \rightarrow \infty} G_{i}\left(t_{i}, u_{n}\left(t_{i}\right)\right) & =G_{i}\left(t_{i}, u\left(t_{i}\right)\right), \quad t \in\left[0, T_{0}\right] .
\end{array}
$$

Therefore from (26), (27), (28), and Lebesgue dominated convergence theorem, we get

$$
\left\|Q u_{n}(t)-Q u(t)\right\| \longrightarrow 0, \quad \text { as } n \longrightarrow \infty,
$$

which implies that $Q$ is continuous on $\left[s_{i}, t_{i+1}\right]$. For $t \in\left[0, t_{1}\right]$, we get

$$
\begin{aligned}
& \left\|\left(\mathcal{Q} u_{n}\right)(t)-(\mathcal{Q} u)(t)\right\| \\
& \quad \leq M \int_{0}^{t}\left\|F\left(\zeta, u_{n}(\zeta)\right)-F(\zeta, u(\zeta))\right\| d \zeta .
\end{aligned}
$$

From the (27), we get

$$
\left\|Q u_{n}(t)-Q u(t)\right\| \longrightarrow 0, \quad \text { as } n \longrightarrow \infty ;
$$

thus, $\mathbb{Q}$ is continuous on $\left[0, t_{1}\right]$. Hence, we conclude that $\mathbb{Q}$ is continuous on $\left[0, T_{0}\right]$.

Secondly, we claim that $\mathbb{Q}\left(B_{k}\right) \subseteq B_{k}$, where $B_{k}(\mathscr{P} \mathscr{C})=$ $B_{k}=\left\{u \in \mathscr{P} \mathscr{C}\left(\left[0, T_{0}\right] ; X\right):\|u\| \leq k\right\}$. For each $u \in B_{k} \subset$ $\mathscr{P} \mathscr{C}\left(\left[0, T_{0}\right] ; X\right)$ and $t \in\left[0, t_{1}\right]$, we get

$\|Q u(t)\|$

$$
\begin{aligned}
& \leq\left\|\mathscr{T}(t) x_{0}\right\|+\int_{0}^{t}\|\mathscr{T}(t-\zeta)\| \cdot\|F(\zeta, u(\zeta))\| d \zeta \\
& \leq M\left\|x_{0}\right\|+M \int_{0}^{t} m_{k}(\zeta) d \zeta \\
& \leq M\left(\left\|x_{0}\right\|+\left\|m_{k}\right\|_{L^{1}\left[0, T_{0}\right]}\right) .
\end{aligned}
$$


For $t \in\left(s_{i}, t_{i+1}\right],(i=1, \ldots, \delta)$, we have

\section{$\|Q \mathbf{Q u}(t)\|$}

$$
\begin{aligned}
& \leq\left\|\mathscr{T}\left(t-s_{i}\right)\right\| \cdot\left\|G_{i}\left(s_{i}, u\left(s_{i}\right)\right)\right\|+M \int_{s_{i}}^{t}\|F(\zeta, u(\zeta))\| d \zeta \\
& \leq M\left(\left\|G_{i}\left(s_{i}, u\left(s_{i}\right)\right)\right\|+\left\|m_{k}\right\|_{L^{1}\left[s_{i}, t_{i+1}\right]}\right) \\
& \leq M\left(\left\|G_{i}\left(s_{i}, u\left(s_{i}\right)\right)\right\|+\left\|m_{k}\right\|_{L^{1}\left[0, T_{0}\right]}\right),
\end{aligned}
$$

which implies that $\|Q \mathcal{Q} u\|_{C\left(\left[0, T_{0}\right], X\right)} \leq M\left(\left\|G_{i}\left(s_{i}, u\left(s_{i}\right)\right)\right\|+\right.$ $\left.\left\|m_{k}\right\|_{L^{1}\left[0, T_{0}\right]}\right)$, for all $i=1, \ldots, \delta$. On the other hand, by the property of $G_{i}(\cdot)$, we get

$$
\|Q \mathcal{Q} u(t)\| \leq\left\|G_{i}(t, u(t))\right\| \leq \sup _{t \in\left(t_{i}, s_{i}\right]}\left\|G_{i}(t, u(t))\right\|,
$$

for $t \in\left(t_{i}, s_{i}\right]$. By the assumption (Hk), we have $M \max _{\{i=1, \ldots, \delta\}}\left[\left\|m_{k}\right\|_{L^{1}\left(\left[0, T_{0}\right]\right)}+\sup _{t \in\left[0, T_{0}\right]}\left\|G_{i}(t, u(t))\right\|+\left\|x_{0}\right\|\right] \leq$ $k$.

Therefore, we conclude that $\|\mathbb{Q} u\|_{\mathscr{P} \mathscr{C}} \leq k$; that is, $\mathbb{Q}$ has values in $B_{k}$.

Now we show the equicontinuity of $Q\left(B_{k}\right)$ on $\left[0, T_{0}\right]$. Since $G_{i}(\cdot)$ is compact, therefore it is obvious that $Q B_{k}$ is equicontinuous on $\left(t_{i}, s_{i}\right]$. Assume $t \in\left[0, t_{1}\right]$. Let $h>0$ be a constant such that $0<t<t+h \leq t_{1}<T_{0}$. For $u \in B_{k}$, we get

$$
\begin{aligned}
\|\mathscr{Q} u(t+h)-\mathscr{Q} u(t)\| \\
\leq\left\|[\mathscr{T}(t+h)-\mathscr{T}(t)] x_{0}\right\| \\
\quad+\| \int_{0}^{t+h} \mathscr{T}(t+h-\zeta) F(\zeta, u(\zeta)) d \zeta \\
\quad-\int_{0}^{t} \mathscr{T}(t-\zeta) F(\zeta, u(\zeta)) d \zeta \| .
\end{aligned}
$$

Using the semigroup property, we have

$$
\begin{aligned}
\| \mathscr{T}( & +h) x_{0}-\mathscr{T}(t) x_{0} \| \\
& =\left\|\mathscr{T}(t)[\mathscr{T}(t+h-t)-\mathscr{T}(0)] x_{0}\right\| \\
& \leq M\left\|[\mathscr{T}(h)-\mathscr{T}(0)] x_{0}\right\| .
\end{aligned}
$$

By the strong continuity of $\mathscr{T}(\cdot)$ and Lemma 14 , we conclude that $Q B_{k}$ is equicontinuous on $\left[0, t_{1}\right]$.

For $t \in\left[s_{i}, t_{i+1}\right]$ we have

$$
\begin{aligned}
\|\mathbb{Q} u(t+h)-\mathscr{Q} u(t)\| \\
\leq\left\|\left[\mathscr{T}\left(t+h-s_{i}\right)-\mathscr{T}\left(t-s_{i}\right)\right] G_{i}\left(s_{i}, u\left(s_{i}\right)\right)\right\| \\
+\| \int_{0}^{t+h} \mathscr{T}(t+h-\zeta) F(\zeta, u(\zeta)) d \zeta \\
\quad-\int_{0}^{t} \mathscr{T}(t-\zeta) F(\zeta, u(\zeta)) d \zeta \| .
\end{aligned}
$$

Since we have

$$
\begin{array}{r}
\left\|\left[\mathscr{T}\left(t+h-s_{i}\right)-\mathscr{T}\left(t-s_{i}\right)\right] G_{i}\left(s_{i}, u\left(s_{i}\right)\right)\right\| \\
\quad \leq M\left\|[\mathscr{T}(h)-\mathscr{T}(0)] G_{i}\left(s_{i}, u\left(s_{i}\right)\right)\right\|,
\end{array}
$$

$G_{i}(\cdot)$ is compact and $\mathscr{T}(\cdot)$ is strongly continuous, which implies that $\mathbb{Q} B_{k}$ is equicontinuous on $\left[s_{i}, t_{i+1}\right]$. Hence, $Q B_{k}$ is equicontinuous on each $\left[0, T_{0}\right]$.

Set $B=\overline{\operatorname{conv}} \mathbb{Q}\left(B_{k}\right)$, where conv and $\overline{c o n v}$ denote the convex hull and closure of the convex hull, respectively. It can be shown easily that $Q$ maps $B$ into itself and $B$ is equicontinuous on each $\left[0, t_{1}\right],\left(t_{i}, s_{i}\right],\left[s_{i}, t_{i+1}\right], i=1,2, \ldots, \delta$. Next we prove that $Q: B \rightarrow B$ is a convex-power condensing operator. We take $u_{0} \in B$ and show that there exists a positive integer $n_{0}$ such that

$$
\beta\left(Q^{\left(n_{0}, u_{0}\right)}(H)\right)<\beta(H),
$$

for every nonprecompact bounded subset $H \subset B$. From (6), (15), and compactness of $G_{i}$, for $t \in\left[s_{i}, t_{i+1}\right]$, where $i=$ $1, \ldots, \delta$, we have

$$
\begin{aligned}
\beta\left(\mathscr{Q}^{\left(1, u_{0}\right)} H(t)\right)= & \beta((\mathscr{Q} H)(t)) \\
\leq & \beta\left(\mathscr{T}\left(t-s_{i}\right) G_{i}\left(s_{i}, H\left(s_{i}\right)\right)\right) \\
& +\beta\left(\int_{s_{i}}^{t} \mathscr{T}(t-\zeta) F(\zeta, H(\zeta)) d \zeta\right) \\
\leq & 4 M \mathscr{L} \int_{s_{i}}^{t} \beta(H(\zeta)) d \zeta \\
\leq & 4 M \mathscr{L}\left(t-s_{i}\right) \beta(H),
\end{aligned}
$$

and similarly for $t \in\left[0, t_{1}\right]$, we have

$$
\beta\left(\widehat{Q}^{\left(1, u_{0}\right)} H(t)\right) \leq 4 M \mathscr{L} t \beta(H) .
$$

For $t \in\left[t_{i}, s_{i}\right]$, we get $\beta\left(\widehat{Q}^{\left(1, u_{0}\right)} H(t)\right)=\beta((Q \mathcal{Q} H)(t))=\beta\left(G_{i}(t\right.$, $H(t)))=0$ by the fact that $G_{i}(\cdot)$ are compact.

Further for $t \in\left[s_{i}, t_{i+1}\right]$, we have

$$
\begin{aligned}
\beta( & \left.\left(\mathscr{Q}^{\left(2, u_{0}\right)} H\right)(t)\right) \\
= & \beta\left(\left(\left(\overline{Q \operatorname{conv}}\left\{Q^{\left(1, u_{0}\right)} H, u_{0}\right\}\right)(t)\right)\right. \\
\leq & \beta\left(\mathscr{T}\left(t-s_{i}\right) G_{i}\left(s_{i}, \overline{\operatorname{conv}}\left\{Q^{\left(1, u_{0}\right)} H, u_{0}\right\}\right)\left(s_{i}\right)\right) \\
& +\beta\left(\int_{s_{i}}^{t} \mathscr{T}(t-\zeta) F\left(\zeta, \overline{\operatorname{conv}}\left\{Q^{\left(1, u_{0}\right)} H, u_{0}\right\}\right)(\zeta)\right) d \zeta \\
\leq & 4 M \int_{s_{i}}^{t} \beta\left(F\left(\zeta, \overline{\operatorname{conv}}\left\{Q^{\left(1, u_{0}\right)} H, u_{0}\right\}\right)\right) d \zeta \\
\leq & 4 M \mathscr{L} \int_{s_{i}}^{t} \beta\left(Q^{\left(1, u_{0}\right)}(H)\right)(\zeta) d \zeta \\
\leq & 4 M \mathscr{L} \int_{s_{i}}^{t} 4 M \mathscr{L}\left(\zeta-s_{i}\right) \beta(H) d \zeta \\
\leq & (4 M \mathscr{L})^{2} \frac{\left(t-s_{i}\right)^{2}}{2} \beta(H) .
\end{aligned}
$$


Proceeding with this iterative method, we get

$$
\beta\left(\left(Q^{\left(n, u_{0}\right)} H\right)(t)\right) \leq \frac{4^{n} M^{n} \mathscr{L}^{n}\left(t-s_{i}\right)^{n}}{n !} \beta(H),
$$

for $t \in\left[s_{i}, t_{i+1}\right]$ and similarly for $t \in\left[0, t_{1}\right]$, we get $\beta\left(\left(Q^{\left(n, u_{0}\right)} H\right)(t)\right) \leq\left(\left(4^{n} M^{n} \mathscr{L}^{n} t^{n}\right) / n !\right) \beta(H)$. Thus, we obtain

$$
\beta\left(\left(\widehat{Q}^{\left(n, u_{0}\right)} H\right)(t)\right) \leq \frac{4^{n} M^{n} \mathscr{L}^{n} T_{0}^{n}}{n !} \beta(H),
$$

for all $t \in\left[0, T_{0}\right]$.

We have that $Q^{\left(n, u_{0}\right)}(H)$ is equicontinuous on $\left[0, T_{0}\right]$ by Lemma 14. Therefore, from Lemma 11, we get

$$
\begin{aligned}
\beta\left(\left(Q^{\left(n, u_{0}\right)} H\right)\right) & =\sup _{t \in\left[0, T_{0}\right]} \beta\left(\left(Q^{\left(n, u_{0}\right)} H\right)(t)\right) \\
& \leq \frac{4^{n} M^{n} \mathscr{L}^{n} T_{0}^{n}}{n !} \beta(H)
\end{aligned}
$$

since we have that $\left(4^{n} M^{n} \mathscr{L}^{n} T_{0}^{n} / n !\right) \rightarrow 0$ as $n \rightarrow \infty$, which infers that there exists a substantial enough positive integer $n_{0}$ such that

$$
\frac{4^{n_{0}} M^{n_{0}} \mathscr{L}^{n_{0}} T_{0}^{n_{0}}}{n_{0} !}<1,
$$

which means that $\mathbb{Q}: B \rightarrow B$ is a convex-power condensing operator. Therefore, from Lemma 10, we get that $Q$ has at least one fixed point in $B$ which is just a mild solution to the problem (1)-(3). This completes the proof of the theorem.

In the next result, the existence of the solution for problem (1)-(3) under Lipschitz conditions of $G_{i}$ is established by using Darbo-Sadovskii's fixed point theorem.

Theorem 16. Suppose that assumptions (H1), (HF), (HG1), and $(H)$ hold. Then, the impulsive problem (1)-(3) has at least one mild solution on $\left[0, T_{0}\right]$.

Proof. Firstly, we decompose the map $\mathbb{Q}$ such that $\mathbb{Q}=$ $\sum_{i=1}^{\delta} Q_{i}^{1}+\sum_{i=1}^{\delta} Q_{i}^{2}$, where $Q_{i}^{j}: \mathscr{P} \mathscr{C}\left(\left[0, T_{0}\right] ; X\right) \rightarrow \mathscr{P} \mathscr{C}([0$, $\left.\left.T_{0}\right] ; X\right), i=0,1,2, \ldots, \delta, j=1,2$, are defined as

$$
\begin{aligned}
Q_{i}^{1} u(t) & \begin{cases}G_{i}(t, u(t)), & \text { for } t \in\left(t_{i}, s_{i}\right], i \geq 1, \\
\mathscr{T}\left(t-s_{i}\right) G_{i}\left(s_{i}, u\left(s_{i}\right)\right), & \text { for } t \in\left(s_{i}, t_{i+1}\right], i \geq 1, \\
0, & \text { for } t \notin\left(t_{i}, t_{i+1}\right], i \geq 0, \\
\mathscr{T}(t) x_{0}, & \text { for } t \in\left[0, t_{1}\right], i=0,\end{cases}
\end{aligned}
$$

$$
\begin{aligned}
& \mathbb{Q}_{i}^{2} u(t) \\
& \quad= \begin{cases}\int_{s_{i}}^{t} \mathscr{T}(t-\zeta) F(\zeta, u(\zeta)) d \zeta, & \text { for } t \in\left(s_{i}, t_{i+1}\right], i \geq 0, \\
0, & \text { for } t \notin\left(s_{i}, t_{i+1}\right], i \geq 0 .\end{cases}
\end{aligned}
$$

For $t \in\left(t_{i}, s_{i}\right]$ and $u, v \in B_{k}$, we have

$$
\begin{aligned}
\| Q_{i}^{1} u & (t)-Q_{i}^{1} v(t) \| \\
& =\sup _{t \in\left[0, T_{0}\right]}\left[\left\|G_{i}(t, u(t))-G_{i}(t, v(t))\right\|\right] \\
& \leq \mathscr{L}_{G_{i}}\|u-v\|_{\mathscr{P} \mathscr{C}},
\end{aligned}
$$

and for $t \in\left(s_{i}, t_{i+1}\right]$

$$
\left\|\widehat{Q}_{i}^{1} u(t)-Q_{i}^{1} v(t)\right\| \leq M \mathscr{L}_{G_{i}}\|u-v\|_{\mathscr{P} \mathscr{C}} ;
$$

therefore, for all $t \in\left[0, T_{0}\right]$ we conclude that

$$
\left\|Q_{i}^{1} u(t)-Q_{i}^{1} v(t)\right\| \leq M \mathscr{L}_{G_{i}}\|u-v\|_{\mathscr{P} \mathscr{C}} .
$$

Since $\beta(H)=\sup _{t \in\left[0, T_{0}\right]}\{\beta(H(t))\}$. By Lemma 5 (vii), we have that for any bounded set $H \subset \mathscr{P} \mathscr{C}$

$$
\beta\left(Q_{i}^{1} H\right) \leq M \mathscr{L}_{G_{i}} \beta(H) .
$$

For operator $\mathbb{Q}_{i}^{2}$, we have

$$
\begin{aligned}
\beta\left(Q_{i}^{2} B_{k}\right) & =\sup _{t \in\left[s_{i}, t_{i+1}\right]} \beta\left(Q_{i}^{2} B_{k}(t)\right) \\
& \leq 2 \sup _{t \in\left[s_{i}, t_{i+1}\right]} \int_{s_{i}}^{t} \beta\left(\mathscr{T}(t-\zeta) F\left(\zeta, B_{k}(\zeta)\right)\right) d \zeta \\
& \leq 4 M \sup _{t \in\left[s_{i}, t_{i+1}\right]} \int_{s_{i}}^{t} \mathscr{L} \beta\left(B_{k}(\zeta)\right) d \zeta \\
& \leq 4 M \mathscr{L}\left(t-s_{i}\right) \beta\left(B_{k}\right) \leq 4 M \mathscr{L} T_{0} \beta\left(B_{k}\right) .
\end{aligned}
$$

From (51)-(53), we have

$$
\begin{aligned}
\beta\left(Q_{k}\right) & \leq \beta\left(Q_{i}^{1} B_{k}\right)+\beta\left(Q_{i}^{2} B_{k}\right) \\
& \leq \max _{\{i=1, \ldots, \delta\}}\left(M \mathscr{L}_{G_{i}}+4 M \mathscr{L} T_{0}\right) \beta\left(B_{k}\right) .
\end{aligned}
$$

From the assumption $(\mathrm{H})$, we get that $\beta\left(Q_{Q} B_{k}\right)<\beta\left(B_{k}\right)$, which implies that map $Q$ is $\beta$-condensing in $B_{k}$. Therefore, by Darbo-Sadovskii's fixed point theorem, the solution map $Q$ has a fixed point in $B_{k}$ which is a mild solution of the nonlocal problem (1)-(3). This completes the proof of the theorem.

\section{Example}

Let us consider the following impulsive problem:

$$
\begin{array}{r}
\frac{\partial}{\partial t} w(t, x)=\frac{\partial^{2}}{\partial x^{2}} w(t, x)+f(t, w(t, x)), \\
(t, x) \in \cup_{i=1}^{N}\left[s_{i}, t_{i+1}\right] \times[0, \pi], \\
w(t, 0)=w(t, \pi)=0, \quad \text { for } t \in\left[0, T_{0}\right], \\
w(0, x)=z(x), \quad x \in[0, \pi]
\end{array}
$$

$w(t, x)=g_{i}(t, w(t, x)), \quad t \in\left(t_{i}, s_{i}\right], x \in[0, \pi]$, 
where $0=t_{0}=s_{0}<t_{1} \leq s_{1}<\cdots<t_{N} \leq s_{N}<t_{N+1}=T_{0}$ are fixed numbers, $z \in X, f \in C\left(\left[0, T_{0}\right] \times \mathbb{R}, \mathbb{R}\right)$, and $g_{i} \in$ $C\left(\left(t_{i}, s_{i}\right] \times \mathbb{R}, \mathbb{R}\right)$, for all $i=1, \ldots, N$.

To convert problem (55) into the abstract form (1)-(3), where $u(t)=w(t, \cdot)$, that is, $u(t) x=w(t, x)$, we introduced the functions $F:\left[0, T_{0}\right] \times X \rightarrow X$ and $G_{i}:\left(t_{i}, s_{i}\right] \times X \rightarrow$ $X$ such that $F(t, u(t))(x)=f(t, w(t, x))$ and $G_{i}(t, u(t))(x)=$ $g_{i}(t, w(t, x))$. We consider $X=L^{2}[0, \pi]$ and the operator $A$ defined as $-A u=u^{\prime \prime}$ with the the domain

$D(A)$

$=\left\{u \in X: u, u^{\prime}\right.$ are absolutely continuous and $u^{\prime \prime} \in X$,

$$
u(0)=u(\pi)=0\} .
$$

It is well known (Pazy [21]) that $-A$ is the infinitesimal generator of an analytic semigroup $\mathscr{T}(t), t \geq 0$ and every analytic semigroup is equicontinuous. This means that $A$ satisfies the assumption (H1).

Next we have that $u \in \mathscr{P} \mathscr{C}\left(\left[0, T_{0}\right] ; X\right)$ is a mild solution of the problem (55).

Case 1. We take

$$
\begin{gathered}
f(t, w(t, x)) \\
=c_{0} \sin |w(t, x)|, \quad t \in\left[0, T_{0}\right], w \in\left[0, T_{0}\right] \times X, \\
g_{i}(t, u(t))(x)=\int_{t_{i}}^{s_{i}} h_{i}(\tau) \log (1+|w(\tau, x)|) d \tau, \\
w \in \mathscr{P} \mathscr{C}\left(\left[0, T_{0}\right] \times X ; X\right), \quad t \in\left[t_{i}, s_{i}\right],
\end{gathered}
$$

and here $h_{i}:\left[s_{i}, t_{i}\right] \rightarrow \mathbb{R}$ are continuous functions and $\int_{t_{i}}^{s_{i}}\left|h_{i}(s)\right| d s<\infty$. Now, we show that $F$ satisfies the assumption (HF). Now, we have that for $u \in X$

$$
\begin{aligned}
\|F(t, u(t))\| & =\left\|c_{0} \sin |(u(t))|\right\| \\
& \leq c_{0}\|u(t)\|,
\end{aligned}
$$

and for $u_{1}, u_{2} \in X$

$$
\begin{aligned}
\| F(t & \left., u_{1}(t)\right)-F\left(t, u_{2}(t)\right) \| \\
& =\left\|c_{0} \sin \left|u_{1}(t)\right|-c_{0} \sin \left|u_{2}(t)\right|\right\| \\
& \leq c_{0}\left[\left\|u_{1}(t)-u_{2}(t)\right\|\right] .
\end{aligned}
$$

Therefore, for any bounded sets $D_{1} \subset X$, we obtain

$$
\beta\left(F\left(t, D_{1}\right)\right) \leq \mathscr{L} \beta\left(D_{1}\right), \quad \text { a.e. } t \in\left[0, T_{0}\right],
$$

where $\mathscr{L}=c_{0}$. For $u \in X$, we have

$$
\begin{aligned}
\left\|G_{i}(t, u(t))\right\| & \leq \int_{s_{i}}^{t_{i}}\left|h_{i}(\tau)\right|\|\log (1+|u(\tau)|)\| d \tau \\
& \leq \int_{s_{i}}^{t_{i}}\left|h_{i}(\tau)\right| d \tau \times\|u(t)\| .
\end{aligned}
$$

Clearly, $G_{i}, i=1, \ldots, \delta$ are compact and satisfy the assumption (HG). Then by Theorem 15, problem (55) has at least a mild solution.

Case 2. Take $f(t, w(t, x))=f_{1}(t) w(t, x)$ and $g_{i}(t, w(t, x))=$ $\left(1 /\left(5+\alpha_{i} t^{3}\right)\right)[|w(t, x)| /(1+|w(t, x)|)], w \in \mathscr{P} \mathscr{C}\left(\left[t_{i}, s_{i}\right] \times X ; X\right) ;$ here, $f_{1}:\left[0, T_{0}\right] \rightarrow \mathbb{R}$ is a bounded and continuous function and $\alpha_{i}>0$. have

For $t \in\left(s_{i}, t_{i+1}\right], i=1, \ldots, \delta$ and $u, u_{1}, u_{2} \in X=\mathbb{R}$, we

$$
\|F(t, u(t)) x\| \leq m_{k}(t)\|u(t)\|,
$$

where $m_{k}(t)=\left|f_{1}(t)\right|$. For any bounded set $D_{1} \subset X$, we have

$$
\beta\left(F\left(t, D_{1}\right)\right) \leq \mathscr{L} \beta\left(D_{1}\right),
$$

where $\mathscr{L}=|f(t)|$. Thus, (HF) holds. For $u_{1}, u_{2} \in \mathscr{P} \mathscr{C}\left(\left[t_{i}\right.\right.$, $\left.\left.s_{i}\right] ; X\right)$, we have

$$
\left\|G_{i}\left(t, u_{1}(t)\right)(x)-G_{i}\left(t, u_{2}(t)\right)(x)\right\| \leq \frac{1}{5}\left\|u_{1}-u_{2}\right\| .
$$

Thus, (HG1) holds. Then by Theorem 16, problem (55) has at least a mild solution.

\section{Conflict of Interests}

The authors declare that there is no conflict of interests regarding the publication of this paper.

\section{Acknowledgments}

The authors would like to thank the referee for valuable comments and suggestions. The work of the first author is supported by the University Grants Commission (UGC), Government of India, New Delhi.

\section{References}

[1] V. Lakshmikantham, D. D. Baĭnov, and P. S. Simeonov, Theory of Impulsive Differential Equations, Series in Modern Applied Mathematics, World Scientific Publishing, Teaneck, NJ, USA, 1989.

[2] M. Benchohra, J. Henderson, and S. Ntouyas, IImpulsive Differential Equations and Inclusions, vol. 2, Hindawi Publishing Corporation, New York, NY, USA, 2006.

[3] Y.-K. Chang, V. Kavitha, and M. M. Arjunan, "Existence results for impulsive neutral differential and integrodifferential equations with nonlocal conditions via fractional operators," Nonlinear Analysis: Hybrid Systems, vol. 4, no. 1, pp. 32-43, 2010.

[4] E. Hernández and D. O’Regan, "On a new class of abstract impulsive differential equations," Proceedings of the American Mathematical Society, vol. 141, no. 5, pp. 1641-1649, 2013.

[5] M. Pierri, D. O’Regan, and V. Rolnik, "Existence of solutions for semi-linear abstract differential equations with not instantaneous impulses," Applied Mathematics and Computation, vol. 219, no. 12, pp. 6743-6749, 2013.

[6] Z. Yan, "Existence of solutions for nonlocal impulsive partial functional integrodifferential equations via fractional operators," Journal of Computational and Applied Mathematics, vol. 235, no. 8, pp. 2252-2262, 2011. 
[7] E. Hernández M., M. Rabello, and H. R. Henríquez, "Existence of solutions for impulsive partial neutral functional differential equations," Journal of Mathematical Analysis and Applications, vol. 331, no. 2, pp. 1135-1158, 2007.

[8] J. H. Liu, "Nonlinear impulsive evolution equations," Dynamics of Continuous, Discrete and Impulsive Systems, vol. 6, no. 1, pp. 77-85, 1999.

[9] J. J. Nieto and R. Rodríguez-López, "Periodic boundary value problem for non-Lipschitzian impulsive functional differential equations," Journal of Mathematical Analysis and Applications, vol. 318, no. 2, pp. 593-610, 2006.

[10] Z. Fan and G. Li, "Existence results for semilinear differential equations with nonlocal and impulsive conditions," Journal of Functional Analysis, vol. 258, no. 5, pp. 1709-1727, 2010.

[11] S. Ji and G. Li, "A unified approach to nonlocal impulsive differential equations with the measure of noncompactness," Advances in Difference Equations, vol. 2012, article 182, 2012.

[12] J. X. Sun and X. Y. Zhang, "A fixed point theorem for convexpower condensing operators and its applications to abstract semilinear evolution equations," Acta Mathematica Sinica. Chinese Series, vol. 48, no. 3, pp. 439-446, 2005.

[13] L. Zhu and G. Li, "Existence results of semilinear differential equations with nonlocal initial conditions in Banach spaces," Nonlinear Analysis: Theory, Methods \& Applications, vol. 74, no. 15, pp. 5133-5140, 2011.

[14] J. Banaś and K. Goebel, Measures of Noncompactness in Banach Spaces, Lecture Notes in Pure and Applied Mathematics, Marcel Dekker, New York, NY, USA, 1980.

[15] R. P. Agarwal, M. Benchohra, and D. Seba, "On the application of measure of noncompactness to the existence of solutions for fractional differential equations," Results in Mathematics, vol. 55, no. 3-4, pp. 221-230, 2009.

[16] P. Chen and Y. Li, "Nonlocal problem for fractional evolution equations of mixed type with the measure of noncompactness," Abstract and Applied Analysis, vol. 2013, Article ID 784816, 12 pages, 2013.

[17] H.-B. Shi, W.-T. Li, and H.-R. Sun, "Existence of mild solutions for abstract mixed type semilinear evolution equations," Turkish Journal of Mathematics, vol. 35, no. 3, pp. 457-472, 2011.

[18] Y. X. Li, "Existence of solutions to initial value problems for abstract semilinear evolution equations," Acta Mathematica Sinica. Chinese Series, vol. 48, no. 6, pp. 1089-1094, 2005.

[19] X. Xue, "Nonlinear differential equations with nonlocal conditions in Banach spaces," Nonlinear Analysis: Theory, Methods \& Applications, vol. 63, no. 4, pp. 575-586, 2005.

[20] X. Xue, "Nonlocal nonlinear differential equations with a measure of noncompactness in Banach spaces," Nonlinear Analysis: Theory, Methods \& Applications, vol. 70, no. 7, pp. 2593-2601, 2009.

[21] A. Pazy, Semigroups of Linear Operators and Applications to Partial Differential Equations, Springer, New York, NY, USA, 1983. 


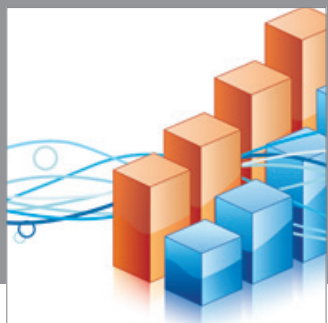

Advances in

Operations Research

mansans

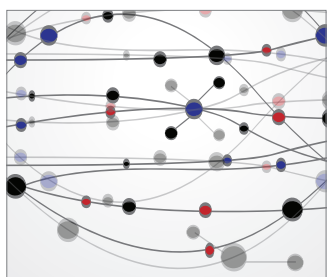

The Scientific World Journal
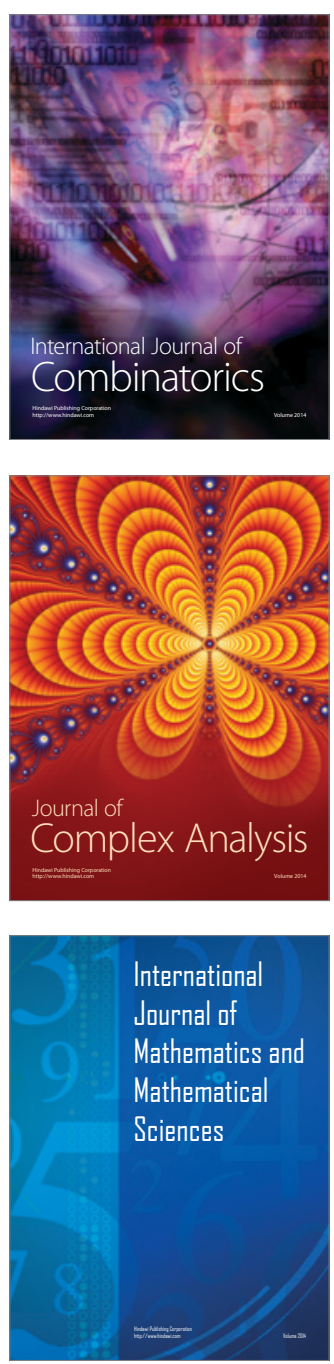
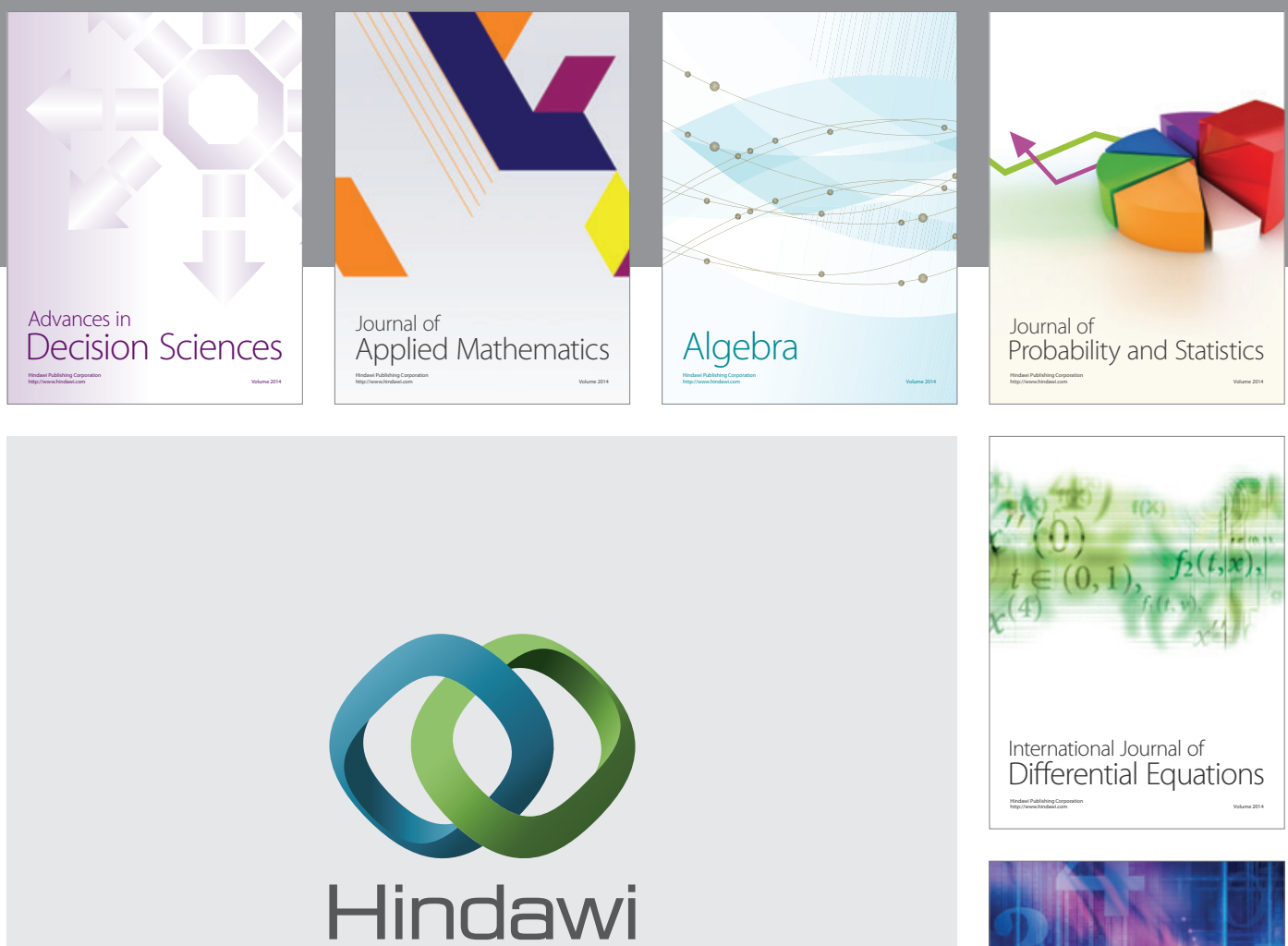

Submit your manuscripts at http://www.hindawi.com
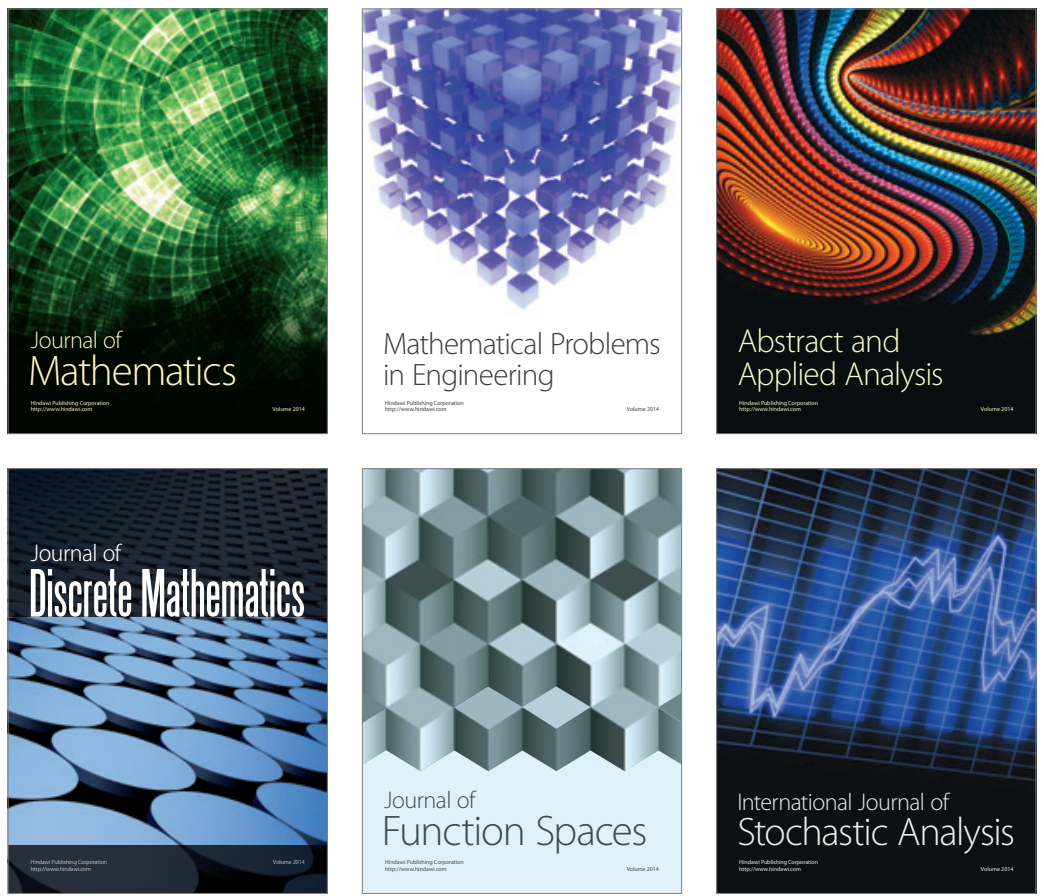

Journal of

Function Spaces

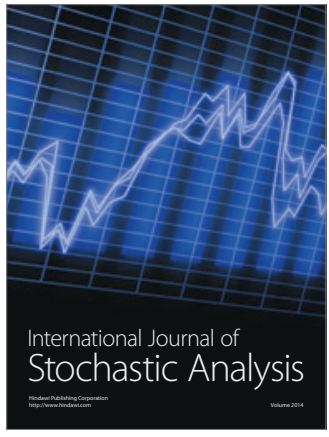

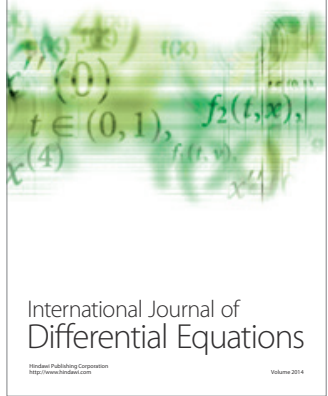
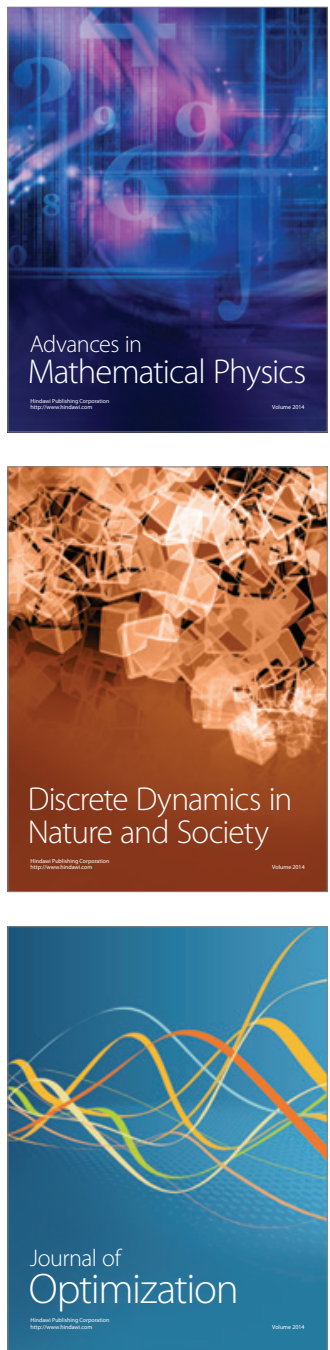